\title{
Enseigner en ligne au moyen d'un wiki : enjeux éthiques et choix technopédagogiques
}

\author{
Marc Couture \\ Professeur, département Science et Technologie \\ TÉLUQ (Université du Québec) \\ marc.couture@teluq.ca
}

Note. Ce texte est le manuscrit final révisé de l'article du même titre paru en mars 2016 dans la revue Éthique publique, vol. 17, n 2, 2015.

\section{Résumé}

Pour un cours universitaire en ligne, j'ai mis en place un wiki calqué sur Wikipédia, reprenant les principes, règles et valeurs de cette communauté. Ainsi, les participants peuvent modifier pratiquement toutes les pages du wiki, même les consignes des activités d'apprentissage. Cependant, lors de la conception du wiki et la mise à l'essai du cours auprès de quelques étudiants, j'ai fait face à un paradoxe à caractère éthique. D'une part, je voulais offrir un environnement de collaboration ouvert et transparent où tous sont soumis aux mêmes règles et tous les enjeux sont abordés au grand jour. D'autre part, mes responsabilités professorales me demandent d'assurer le respect des objectifs du cours, d'appliquer divers règlements, de garantir l'intégrité de l'évaluation et de maintenir des conditions propices à l'apprentissage. Cette tension m'a amené à une série de compromis, notamment sur la transparence des interactions professeur-étudiants. La question se pose alors : dans quelle mesure les valeurs et pratiques des communs peuvent-elles coexister avec les contraintes propres à un contexte formel d'apprentissage?

\section{Mots-clés}

Wiki, enseignement, apprentissage, éthique, communs

\begin{abstract}
For an online university course, I devised a Wikipedia-like wiki based upon the same principles, rules and values. For instance, users may edit almost any page, even the learning activities instructions. However, during the design phase and the ensuing test period involving a few students, I faced a paradox of ethical nature. On the one hand, I wanted to provide an open and transparent collaborative environment, where everybody abides by the same rules and all issues are discussed overtly. On the other hand, as part of my responsibilities as a professor, I must respect course objectives, enforce administrative rules, ensure the integrity of assessment, and maintain conditions which facilitate learning. This tension led to various compromises, notably on the transparency of student-teacher interactions. The question is still open: To what extent is it possible to apply the values and practices of the commons to a formal learning environment?
\end{abstract}

\section{Keywords}

Wiki, teaching, learning, ethics, commons 


\section{INTRODUCTION}

Le cours en ligne de premier cycle SCI 1014 La science ouverte est offert à distance depuis l'automne 2014. Il s'adresse en premier lieu aux étudiants inscrits dans un des programmes généraux de science de la TÉLUQ, mais toute personne détenant une bonne connaissance de la recherche scientifique peut s'y inscrire. Le sujet du cours et ses objectifs, de même que ceux des programmes dont il fait partie, rendaient particulièrement pertinente l'utilisation d'un wiki s'inspirant fortement de Wikipédia, à la fois comme environnement informatique d'apprentissage et outil de structuration des actions et des interventions des étudiants.

Après un bref survol des travaux sur le fonctionnement de Wikipédia et l'usage des wikis (dont Wikipédia) en enseignement, je décrirai la démarche pédagogique du cours SCI 1014 et son wiki ${ }^{1}$, en mettant l'accent sur ce qui le rapproche, ou le différencie de Wikipédia. Je présenterai ensuite un certain nombre de décisions faisant intervenir des considérations éthiques que j'ai dû prendre, à titre de responsable du cours, au sujet des caractéristiques du wiki et des règles de fonctionnement destinées aux étudiants et au professeur. Les choix possibles ne répondaient pas uniquement à des considérations éthiques : ils étaient aussi soumis aux lois et aux règlements internes, notamment en matière de protection des renseignements personnels, de même qu'à des exigences pédagogiques et administratives, au premier plan celle d'évaluer de manière équitable l'atteinte des objectifs du cours par chacun des étudiants. J'insisterai sur la tension entre les rôles que joue le professeur, responsable à la fois d'assurer le respect de ces exigences et d'offrir aux participants, certains inscrits au cours et d'autres non, une expérience authentique de collaboration ouverte. Finalement, j'expliquerai comment la dynamique d'un tel wiki, bien que contrainte par ces exigences pédagogiques et réglementaires, s'inscrit dans celle que l'on associe généralement aux communs.

Je tiens à préciser que le point de vue que je présente ici est exclusivement celui du responsable du cours, qui l'a conçu et qui a encadré les étudiants durant sa mise à l'essai.

\section{WIKI(PÉDIA) ET L'ENSEIGNEMENT}

Les valeurs et les principes de base de Wikipédia peuvent être déduits d'un certain nombre de documents inclus dans l'encyclopédie, notamment l'énoncé de principe de son fondateur, Jimmy Wales (2001), et certaines pages qui la décrivent de manière globale, telles «Wikipédia:Principes fondateurs » et «Wikipedia:Expectations and norms of the Wikipedia community ».

On peut les résumer ainsi (les citations en italique sont tirées de Wales (2001) :

- L'ouverture. Tous peuvent consulter et modifier l'encyclopédie sans formalités ni contrepartie, telle l'inscription avec fourniture de renseignements personnels ( «You can edit this page right now »), et tous peuvent en réutiliser le contenu, sans autres contraintes que celles de la licence CC BY-SA.

1. http://wiki.teluq.ca/SCI1014 
- L'égalité. Tous les contributeurs de Wikipédia, du novice au plus expérimenté, ont les mêmes droits et sont soumis aux mêmes règles («There must be no cabal, there must be no elites, there must be no hierarchy or structure which gets in the way of this openness to newcomers $\gg$ ).

- La transparence. Tout ce qui est rédigé dans le wiki est visible de tous et le demeure indéfiniment.

- La collaboration. Les contributeurs fondent leurs actions et leurs décisions sur un même objectif : travailler avec les autres à l'amélioration de l'encyclopédie, dans un esprit de respect et de civisme.

- La participation aux décisions. Toute décision est précédée d'une discussion, dans laquelle tous sont conviés à fournir leur point de vue, visant l'atteinte d'un consensus.

De nombreuses études portant sur le fonctionnement réel de la communauté Wikipédia (voir par exemple Cardon et Levrel, 2009; Jacquemin, 2012; O’Neil, 2011; Reagle, 2011) suggèrent que ces principes ne s'incarnent pas toujours de manière stricte. En particulier, l'égalité de tous et l'absence de hiérarchie sont en partie des vues de l'esprit. En effet, même si ce n'est pas apparent, surtout pour les novices $(\mathrm{Oz}, 2009)$, on y trouve une structure administrative très élaborée. Celle-ci peut être informelle, comme la « patrouille RC », qu'on peut joindre de sa propre initiative, qui surveille les changements récents. Elle peut aussi être plus formelle, comme le groupe des administrateurs, élus et agissant après consultation. Se fondant sur des règles complexes, ils peuvent bloquer des pages et des utilisateurs (ce qui va à l'encontre du principe d'ouverture); ils peuvent aussi effacer des pages et leur historique (non-transparence). Cela a suffi pour que certains, réfutant le mythe de son « ouverture radicale », proclament, la « mort de Wikipédia » (Carr, 2011). Sans aller aussi loin, on peut certainement remettre en question la vision idéaliste de Wikipédia comme environnement où tous sont égaux et peuvent faire ce qu'ils veulent en toute liberté, du moment qu'ils estiment « agir correctement » («do the right thing »), c'est-à-dire améliorer l'encyclopédie et respecter les autres (Lichtenstein, 2008). Mais plusieurs voient dans ce qui présente parfois l'allure d'une bureaucratie au fonctionnement fastidieux, à certains égards opaque, l'explication même de la capacité de Wikipédia à maintenir sa qualité malgré les actions des «vandales » ou autres délinquants qui tirent profit de son ouverture (Grimmelmann, 2015 : 79-87).

Les wikis, qu'ils s'approchent ou non du modèle Wikipédia, n'ont pas tardé à trouver leur place dans l'enseignement (Fountain, 2005; Zheng, Niiya et Warschauer, 2015). Dans la plupart des cas, le wiki forme l'une des composantes d'un cours, qui comprend également des activités en classe, où les étudiants se rencontrent physiquement. Dans certains cas, notamment dans les cours entièrement en ligne, il constitue un élément du système de gestion des contenus (content management system, ou CMS). Le wiki est alors employé pour la réalisation d'une ou de quelques-unes des activités d'apprentissage.

Ces wikis se distinguent par leur accessibilité. Certains sont privés, c'est-à-dire réservés, en lecture comme en écriture, aux étudiants inscrits au cours à un trimestre donné. D'autres, dits semi-publics, sont ouverts, en lecture et (ou) en écriture, à une communauté plus large, par exemple l'ensemble des étudiants d'un établissement (Bachelet, 2008), voire à tous les internautes (Schneider, Benetos et Ruchat, 2011). Ils se distinguent aussi par la marge de 
manœuvre laissée aux étudiants (Moshirnia, 2007). Certains n'imposent que le sujet du wiki et des objectifs généraux, laissant les étudiants décider de quelle façon ils exploiteront les fonctions du wiki et en détermineront le contenu. D'autres proposent un encadrement plus serré, soit par l'imposition de modalités (par exemple le travail en équipe, ou des échéances préétablies), soit par le truchement de l'évaluation, en attribuant des points à diverses tâches que les étudiants sont invités à réaliser.

\section{LE COURS SCI 1014 ET SON WIKI}

\section{La démarche pédagogique du cours}

Les étudiants peuvent s'inscrire et commencer le cours en tout temps; ils disposent alors de 15 semaines pour le terminer. Une feuille de route propose une répartition des activités d'apprentissage sur ces 15 semaines, mais les étudiants sont libres d'aménager leur calendrier à leur guise. Ainsi, des étudiants qui en sont à divers stades dans le cours sont actifs en même temps dans le wiki.

Les activités d'apprentissage du cours sont les suivantes.

- Deux activités non notées : une activité de démarrage et une activité d'exploration, où les étudiants prennent connaissance du fonctionnement du wiki et des textes de base.

- Quatre travaux notés : la rédaction d'une ébauche, d'un article, l'amélioration d'un texte déjà présent dans le wiki et la discussion d'un second texte en vue de guider son amélioration ultérieure par d'autres étudiants.

- Un journal de bord, où les étudiants consignent leurs contributions au fonctionnement du wiki et leurs réflexions sur un certain nombre d'enjeux éthiques liés à la science ouverte, et où ils effectuent un bilan (noté) de ces réflexions et de leur participation au wiki.

\section{Le wiki du cours}

Le choix d'un wiki s'inspirant fortement de Wikipédia s'imposait de manière naturelle compte tenu du sujet du cours, ainsi que des objectifs du cours et des programmes dont il fait partie, qui incluent la compréhension du caractère novateur des pratiques de la science ouverte et le développement de compétences liées à l'intervention et à la communication dans des milieux scientifiques ou plus larges. D'une part, on peut définir la science ouverte comme l'application des outils et pratiques du web social à l'ensemble des étapes de la recherche scientifique, avec comme objectifs premiers la transparence et la collaboration (Mogin, 2010; Nielsen, 2011). Or, parmi les environnements informatiques du web social, les wikis comptent parmi ceux qui incarnent le mieux, surtout si leur degré d'ouverture et de transparence se rapproche de celui de Wikipédia, la transformation des valeurs, des attitudes et des pratiques qui caractérise la science ouverte. D'autre part, bien que ne pouvant soutenir des travaux de recherche originaux («Wikipédia:Travaux inédits »), Wikipédia constitue un excellent outil pour le partage des résultats des recherches avec un large public.

Sur un plan opérationnel, cela s'est traduit par le choix du même logiciel (MediaWiki) que l'encyclopédie, et la reprise de la plupart des règles et recommandations s'appliquant à la contribution à Wikipédia, tant en ce qui touche l'écriture (formats, liens, citations, notes et 
références, etc.) que la communication entre utilisateurs (page utilisateur, pages de discussion). Ces choix visaient également à préparer les étudiants à intervenir efficacement dans Wikipédia par la suite.

Par contre, quelques caractéristiques importantes de Wikipédia n’ont pas été retenues :

- la neutralité de point de vue;

- l'interdiction des travaux inédits;

- la participation universelle : toute décision se prend au moyen d'un vote ou d'une recherche de consensus, auxquels tous peuvent participer;

- la transparence totale : tous les textes hébergés dans Wikipédia, y compris les discussions au sein des groupes restreints (par exemple, les administrateurs), sont accessibles à tous.

Les deux premières, justifiées essentiellement par le caractère encyclopédique de Wikipédia, ont été écartées pour une raison simple : le wiki de SCI 1014 ne vise pas à construire une encyclopédie de la science ouverte. Les deux autres l'ont été pour des considérations en partie éthiques, discutées plus loin.

Sur le plan de la structure, le wiki de SCI 1014 comprend une douzaine de rubriques, accessibles à partir de toute page et regroupées en trois catégories.

- INFORMATIONS : liste des travaux effectués par les étudiants; liste des participants, liste des modifications récentes.

- CONTENU : présentation du cours; consignes des activités d'apprentissage et des travaux notés (incluant les critères d'évaluation); textes de base (un pour chacune des cinq dimensions de la science ouverte retenues), articles rédigés ou modifiés par les étudiants; webographie.

- ASSISTANCE : pages d'aide et guides, la plupart adaptés des pages d'aide de Wikipédia; café étudiant (lieu de discussions générales); code de conduite, s'appliquant aux étudiants et au professeur.

Il contient également quelques pages consacrées au droit d'auteur, à l'utilisation dans le wiki de contenu externe et à la réutilisation à l'externe de son contenu.

Ce wiki se démarque de la plupart des wikis éducatifs mentionnés dans la littérature en ce qu'il constitue l'unique plateforme employée pour le cours. Ainsi, l'ensemble des informations et consignes nécessaires aux étudiants pour qu'ils puissent suivre le cours de manière autonome se retrouve dans des pages du wiki, au même titre que celles que les étudiants sont appelés à créer et à améliorer dans leurs activités. De même, le wiki (principalement les pages de discussion, comme dans Wikipédia) est l'outil suggéré pour la plupart des interactions entre les étudiants ou entre ceux-ci et le professeur.

\section{DES ENJEUX À CARACTÈRE ÉTHIQUE}

Du début de la conception du cours à sa mise à l'essai, j'ai dû prendre diverses décisions faisant intervenir des enjeux éthiques, souvent en même temps que des enjeux pédagogiques ou administratifs (Fountain, 2005). Certains de ces enjeux sont d'ailleurs proposés aux étudiants pour leur réflexion éthique (journal de bord). 
Ces décisions portaient sur les dimensions suivantes :

- la visibilité et l'accessibilité du wiki;

- l'anonymat et la confidentialité;

- le rôle du professeur et ses interventions auprès des étudiants.

\section{Visibilité et accessibilité}

Un premier enjeu, lié à l'ouverture, du wiki, concerne son accessibilité (en écriture) et sa visibilité (en lecture). Les études sur les wikis utilisés dans des cours (Baltzersen, 2010; Every, Garcia et Young, 2010; Guth, 2007) ont montré, d'une part, que les contributions de l'extérieur (quand elles sont permises) sont très rares et, d'autre part, que rendre les travaux des étudiants largement accessibles constitue une source de motivation, et les pousse à y porter plus de soin. Par ailleurs, le pollupostage (spam) demeure une préoccupation pour tout environnement largement ouvert en écriture.

J'ai décidé de rendre le wiki de SCI 1014 visible pour tous les internautes. De son côté, l'accès en écriture est partiellement limité; outre les étudiants du cours, tous les membres de la communauté universitaire (étudiants et personnel) peuvent contribuer au wiki. Comme tous les étudiants inscrits à la TÉLUQ, même pour un seul cours, conservent leur accès pendant deux ans, on parle ici d'un potentiel de plus de 10000 contributeurs. Ce choix visait entre autres à permettre aux étudiants ayant suivi le cours de continuer à contribuer au wiki après la fin de celui-ci.

La dimension éthique de cette décision porte sur la responsabilité du professeur à l'égard du contenu associé à son propre nom et portant la marque de l'université. En effet, les règles d'évaluation de la crédibilité des documents en ligne (voir par exemple Université du Québec à Montréal, n.d.) accordent à un site universitaire une crédibilité élevée, celle-ci reposant en bonne partie sur la compétence du ou des professeurs concernés. De plus, à la TÉLUQ, on effectue généralement une évaluation du contenu des cours avant leur offre publique. En dépit de la présence dans le wiki d'une page d'avertissement, d'ailleurs assez floue sur la manière exacte dont le professeur serait garant de la qualité du contenu (on y affirme qu'il « suit de près le wiki »), il ne sera sans doute pas évident pour les visiteurs que le contenu n'est pas soumis aux mêmes normes de qualité que les cours de la TÉLUQ ou les textes normalement rédigés par le professeur. Ce risque est quelque peu tempéré par la décision de permettre aux étudiants, bien que cela aille à l'encontre du principe de transparence, de soumettre hors wiki une première version de la plupart des travaux notés.

Il fallait ensuite déterminer si toutes les pages du wiki peuvent être modifiées. Dans Wikipédia, presque toutes les pages sont accessibles en écriture de façon permanente. Outre des pages qui ne doivent pas être modifiées pour des considérations techniques ou administratives, seules la page d'accueil et cinq pages d'aide (parmi des centaines) s'adressant aux débutants sont bloquées en écriture de manière permanente. Je souhaitais appliquer la même ouverture au wiki de SCI 1014.

Pour la plupart des textes, que j'avais rédigés seul au départ, l'enjeu éthique concerne essentiellement la relation que j'entretiens avec eux, et touche directement mon double rôle de professeur et de contributeur au wiki (voir plus loin « Rôles et interventions du professeur »). Pour les pages de la rubrique Activités, l'enjeu est très différent : on touche ici à la responsabilité du professeur d'offrir aux étudiants les moyens d'atteindre les objectifs 
du cours, et d'attester de cette atteinte par l'octroi d'une note individuelle. Les étudiants peuvent modifier la formulation des consignes, pour les clarifier ou les préciser. Cependant, ils ne peuvent modifier ni les critères d'évaluation, qui précisent notamment l'ampleur de chacune des activités, ni leur pondération, ni certaines exigences, par exemple en ce qui concerne les sources documentaires. Ils ne peuvent non plus modifier le nombre et les titres des activités. On notera que cela n'empêche nullement les étudiants d'exprimer, dans les pages de discussion de cette rubrique, leurs commentaires et suggestions sur tous les aspects des activités, y compris les critères d'évaluation et la pondération.

\section{Conditions d'utilisation}

Un second enjeu, lié aussi à l'ouverture, porte sur le choix de la licence d'utilisation des textes du wiki. Selon le paradigme du libre accès, une des composantes de la science ouverte, l' « ouverture » ne se limite pas à l'accès gratuit, mais comprend l'octroi au public d'une liberté dans la réutilisation des œuvres. Cet octroi se manifeste par la diffusion sous licence utilisateur, les licences Creative Commons étant vues comme les plus appropriées. En dépit de certaines réserves (Morrisson, 2012 : 49-61), il est généralement admis dans le monde de l'accès libre que la licence accordant la plus grande liberté, soit CC BY (attribution) est celle qui convient le mieux, en raison de la quasi-absence de contraintes et d'ambiguïtés (Carroll, 2013). C'est la licence que je souhaitais employer. Cependant, des considérations pratiques, liées à l'intérêt de pouvoir intégrer dans le wiki des contenus provenant de Wikipédia (textes) et WikiCommons (images), a fait pencher la balance en faveur de la même licence que ceux-ci, soit CC BY-SA. Cette licence oblige à diffuser sous les mêmes conditions, donc avec la même licence, toute œuvre réutilisant en tout ou en partie l'œuvre originale.

Une des dimensions éthiques de cette décision, quelle que soit la licence choisie, est que son usage est imposé aux étudiants; cet enjeu est encore plus délicat dans le contexte où le cours est obligatoire pour certains. Des considérations juridiques interviennent également ici, car une telle exigence pourrait être considérée comme une clause abusive, donc éminemment contestable, du « contrat d'adhésion » que constitue l'inscription au programme ou au cours. Mais cette exigence peut aussi être vue comme une simple question de cohérence, compte tenu du sujet du cours, ce qui semble partagé par les commentaires des étudiants à ce sujet dans l'activité « Journal de bord ». Quoi qu'il en soit, les étudiants doivent en être très clairement informés au moment de choisir le cours; ceux pour qui il est obligatoire devraient pouvoir choisir un autre cours pour répondre aux exigences du programme. Le wiki EduTech (un wiki éducatif qui présente de nombreuses similarités avec celui de SCI 1014) offre à ceux qui le souhaitent de diffuser « leur» texte, sous des conditions différentes de celle de la licence générale, en l'indiquant dans la page ( «EduTech_Wiki:Copyrights»). Cette solution est cependant susceptible d'entraîner une certaine confusion quant à ce qu'il est permis de réutiliser, et pourrait entrer en conflit avec les souhaits des étudiants modifiant la page par la suite.

\section{Anonymat et confidentialité}

Un troisième enjeu à caractère éthique, lié à la transparence, est présent dans tous les environnements de collaboration (wikis, blogues, sites questions/réponses, etc.); il touche l'anonymat et la confidentialité. Certains environnements associés à la science ouverte, comme ResearchGate, exigent que les participants s'affichent sous leur propre nom. Mais la 
plupart offrent le choix aux utilisateurs, qui emploient des formes allant du nom complet ou partiel à des pseudonymes sibyllins.

L'anonymat est à double tranchant. D'une part, il permet une plus grande liberté d'expression. On songe souvent aux résidents de pays à régime autocratique, mais on peut aussi penser aux employés qui remettraient en question des pratiques discutables dans leur organisation. D'autre part, l'anonymat peut déresponsabiliser les utilisateurs, qui n'ont pas à assumer publiquement leurs écrits et leurs actions, du moins face aux autres utilisateurs (Santana et Wood, 2009). Dans le wiki de SCI 1014, les étudiants et le personnel de la TÉLUQ sont identifiés par leur nom d'usager, un code à 8 caractères. Pour le personnel, celui-ci combine le nom (ou son début) et l'initiale du prénom, ce qui élimine l'anonymat à toutes fins utiles. De son côté, le code des étudiants est formé de «Et » suivi de 6 chiffres; un étudiant a le même code dans tous les environnements en ligne de la TÉLUQ (sites de cours, forums, etc.).

J'ai décidé de laisser les étudiants entièrement libres à cet égard, tant dans les commentaires qu'ils inscrivent que dans leur page utilisateur, où ils se présentent. En pratique, ils emploient souvent le prénom et l'initiale de leur nom de famille. Il s'agit donc d'un anonymat à géométrie variable (Flinn et Maurer, 1995) : s'ils ne dévoilent pas leur nom complet, ils sont véritablement anonymes pour les visiteurs de l'extérieur, mais semianonymes pour les membres de la TÉLUQ, qui auraient pu lire leurs interventions sous la même identité dans d'autres environnements de l'université, et pas du tout anonymes pour l'établissement : le professeur connaît l'identité des étudiants inscrits au cours; celle des autres étudiants est accessible à certains membres du personnel. Une des conséquences de cette situation est que, en vertu de la loi sur la protection des renseignements personnels, je dois veiller à ne fournir aucune information qui pourrait permettre d'identifier un étudiant, même indirectement, par recoupement par exemple. Cela n'est pas toujours évident, comme je l'ai constaté en rédigeant un commentaire mentionnant l'étudiant EtXXXXXX, qui n'avait encore rien inscrit dans le wiki : j'avais choisi, de manière humoristique et pour éviter l'emploi d'un code aussi rébarbatif, de le nommer « monsieur X », dévoilant ainsi son sexe.

\section{Rôle et interventions du professeur}

Outre ces enjeux touchant les principes d'ouverture et de transparence, de même qu'au lien qu'entretient un auteur avec son œuvre, trois enjeux éthiques majeurs portent sur des décisions en matière d'interventions du professeur en réaction aux contributions et, le cas échéant, aux comportements des étudiants dans le wiki.

Le premier, qui concerne uniquement les étudiants inscrits au cours, touche la confidentialité de l'évaluation. Toutes les contributions des étudiants sont sujettes à évaluation (et à notation). Or, au même titre que son identité, la note d'un étudiant est une information confidentielle, qui ne serait pas protégée par l'anonymat tout relatif régnant dans le wiki. Les choses sont moins claires pour les commentaires du correcteur sur les travaux, qui justifient la note accordée et à partir desquels on peut se faire à tout le moins une idée de la note obtenue (par exemple, « vous êtes passé complètement à côté du sujet »). Et au-delà de la réglementation, se profile l'enjeu plus proprement éthique du respect des personnes. Tout comme les gestes d'humiliation (commentaires publics peu flatteurs, « bonnet d'âne ») ont été depuis longtemps évacués des pratiques éducatives, on peut s'interroger sur la pertinence 
de rendre publics ces commentaires, même formulés de manière constructive (par exemple : « vous auriez intérêt à revoir en profondeur la structure et la formulation de votre texte »). Une première décision a été de traiter de manière confidentielle une première version des activités de rédaction et d'amélioration d'articles : dans un premier temps, les étudiants qui rédigent ou modifient un article le font discrètement, en dehors du wiki, ou à tout le moins en dehors des pages « officielles » qui hébergent les textes. La rétroaction du professeur, qui ne contient pas de note à ce stade, est transmise de manière confidentielle. C'est une version révisée, placée celle-là dans une page officielle du wiki, qui est sujette à notation, communiquée de manière confidentielle bien entendu. J'ai cependant décidé de placer dans la page de discussion de l'article ma rétroaction à cette version révisée, de manière à offrir des lignes directrices aux étudiants appelés plus tard à améliorer ce texte. Je prends cependant soin de rédiger ma rétroaction de la manière la plus constructive et respectueuse possible, mais il est clair qu'on peut y déceler des indices de la « valeur » du travail de cet étudiant aux yeux du professeur.

De plus, les articles sont classés, par un bandeau en début de page, selon trois niveaux d'avancement s'inspirant fortement de ceux de Wikipédia (« Modèle:Tableau d'évaluation avancement »). Ceux-ci sont liés notamment à leur ampleur et à leur contenu : lien avec le sujet du cours, couverture suffisante du sujet, structure, nombre et qualité des références. Dans Wikipédia, l'attribution de ces niveaux fait l'objet, comme pour toute décision, de discussions, voire de votes formels, auxquels participent les personnes regroupées autour de « projets ». Dans le cours SCI 1014, les activités ont pour résultat soit la création d'un article de niveau un ou trois, soit l'amélioration d'un article pour le faire progresser d'un niveau (s'il est à l'un des deux premiers). À l'origine, les étudiants devaient modifier euxmêmes le bandeau d'avancement en conséquence. Durant la mise à l'essai, j'ai fait face au problème d'articles qui, même après révision, n'atteignaient pas le niveau prévu. J'ai alors pris la décision de gérer l'attribution des niveaux, après la correction, et d'ajouter des « demi-niveaux », qui signifient que le texte doit être retravaillé, plutôt qu'enrichi, pour passer au niveau suivant. Cette information qui, encore plus que les commentaires, se rapproche d'une note (de passage) est pourtant essentielle pour permettre aux étudiants de savoir ce qui est attendu d'eux quand ils décident d'améliorer un article.

Le second enjeu concerne le rôle du professeur comme contributeur au wiki. Avant même que le cours reçoive ses premiers étudiants, les rubriques du wiki étaient déjà bien garnies, $\mathrm{y}$ compris celle devant regrouper les textes des étudiants. Celle-ci contenait quatre ébauches de mon cru, afin que les premiers étudiants puissent modifier des textes de même niveau que ceux que d'autres étudiants auraient normalement produits plus tôt. J'étais donc l'auteur (unique dans la plupart des cas) de l'entièreté des textes de départ, dont tous étaient susceptibles d'être modifiés par les étudiants (les ébauches, à coup sûr). Je me retrouvais alors face au même enjeu auquel sont soumis les étudiants et sur lequel ils sont appelés à réfléchir dans leur journal de bord : la nécessité d'établir une distance, ou un détachement, par rapport à son propre texte. Celui-ci devient en effet rapidement un texte collectif dont, dès lors, la responsabilité n'incombe plus, ou plus exclusivement à son auteur initial. Cet enjeu se manifeste pour moi de manière cruciale au moment de la rétroaction : je peux être appelé à juger de la pertinence de modifications ou d'ajouts à mes textes. Cela est susceptible de remettre en question des choix reflétant mes convictions, fondées sur mes compétences et mes connaissances en rapport avec le sujet du cours, mais que d'autres pourraient bien considérer comme des préférences personnelles. En quelque sorte, je me 
trouve ici en situation de conflit d'intérêts, qu'il me faut gérer. Dans mon rôle de professeur, je dois faire preuve de bonne foi et d'humilité dans mon évaluation des ajouts et modifications qu'apportent les étudiants. Je dois aussi réévaluer quels sont mes intérêts réels à l'égard d'une éventuelle diminution (de mon point de vue d'auteur initial) de la qualité du wiki. En d'autres termes, à quel point puis-je, ou dois-je être garant de la qualité du wiki? Dans mon rôle de contributeur au wiki, j'ai finalement décidé de me limiter à des suggestions d'amélioration, dans les pages de discussion des articles, et à des corrections de forme (langue, format du texte, notices bibliographiques, etc.) dans les articles eux-mêmes.

Finalement, un troisième enjeu touche la résolution de certains problèmes, comme le vandalisme ou les différends, voire les conflits, pouvant survenir dans le wiki. À cet égard, il faut mentionner que des politiques ou procédures s'appliquent à tous les étudiants, quel que soit le cours, en matière de révision de note, de traitement des plaintes, de plagiat, de fraude et de comportement répréhensible. Ces politiques « externes » pourraient éventuellement suffire, mais en raison de leur lourdeur, et pour conserver une partie du principe d'autogestion qui caractérise Wikipédia, j'ai prévu une procédure interne pour désamorcer les problèmes avant qu'ils ne dégénèrent. Comme dans Wikipédia, une personne qui détient les droits d'administrateur (voir plus haut), avant de poser une action, cherchera à obtenir le point de vue des participants, lorsque la chose est possible; elle expliquera clairement les raisons qui justifient sa décision. C'est le professeur qui joue ce rôle, sauf si lui-même ou un étudiant qui suit le cours est directement concerné. En effet, toute décision en faveur de cet étudiant entrerait en conflit avec l'exigence non seulement d'une équité de traitement et d'évaluation pour tous, mais de la perception de la présence de cette équité. Dans ce cas, j'ai désigné une personne qui ne contribue pas au wiki, mais qui est très familière avec ce type d'environnement, pour recevoir et traiter toute demande ou plainte impliquant le professeur ou un étudiant qui suit le cours.

\section{LE WIKI DE SCI 1014 EST-IL UN COMMUN?}

Compte tenu du contexte dans lequel s'inscrivent les interventions des participants, en particulier les étudiants du cours, ainsi que du rôle du professeur, qui détient une autorité à la fois morale et administrative, à quel point le wiki de SCI 1014 s'éloigne-t-il de ce qui fait de Wikipédia un véritable commun, c'est-à-dire une ressource partagée par une communauté, qui en empêche la privatisation et la gère pour le bien de tous grâce à « un ensemble de pratiques, valeurs et normes sociales » (Bollier, 2014 : 29).

Dans Wikipédia comme dans le wiki de SCI 1014, on est en présence d'une ressource, soit une série de textes et de consignes visant à guider les contributions, que l'on peut qualifier de «partagée » dans la mesure où tout participant peut la modifier, et aucun n'a de contrôle sur le destin de ses propres contributions. Celles-ci peuvent non seulement être modifiées par d'autres participants, mais aussi, en dehors du wiki, par tout internaute qui réutilise les textes en vertu de la licence Creative Commons. Il faut réaliser à quel point cela contraste avec la situation qui règne dans un cours traditionnel. Les travaux d'évaluation y sont la propriété exclusive de l'étudiant, même si le professeur, par ses commentaires, a pu l'aider à en accroître la qualité, donc la «valeur ». Cette privatisation se traduit, par exemple, par le fait qu'un étudiant peut «prêter » un travail à un autre, voire le vendre (des pratiques ni illégales, ni proscrites par les règlements d'éthique, soit dit en passant). La seule différence à cet égard entre Wikipédia et le wiki du cours est que, dans celui-ci, ce n'est pas l'ensemble 
des internautes, mais un groupe restreint, bien que plus large que les seuls inscrits au cours, qui peut modifier le contenu du wiki, du moins à l'intérieur de celui-ci.

L'autre dimension des communs est la présence - et leur gestion par la communauté - de règles qui incarnent ou encadrent des pratiques, des valeurs et des normes sociales. Comme cela a été expliqué, ces règles sont à peu près les mêmes dans les deux wikis; elles incluent notamment des consignes et critères précisant le type de texte acceptable ou souhaitable, de même que leur structure et leur contenu. Dans Wikipédia, elles répondent à l'objectif de produire une encyclopédie; dans le wiki du cours, aux objectifs d'apprentissage. Dans les deux wikis également, les participants peuvent modifier les textes énonçant ces règles, à l'exception, dans le wiki du cours, des critères et des barèmes d'évaluation.

On peut tout de même observer ici trois différences significatives. Premièrement, contrairement à celles de Wikipédia, les règles du wiki du cours ont été imposées au départ par le professeur; elles n'ont pas émergé de la communauté. Deuxièmement, on peut penser que les étudiants ne se sentent pas réellement libres de modifier les consignes, soit parce qu'ils craignent de déplaire au professeur et de voir leur évaluation en être affectée, soit parce qu'ils pensent que celui-ci va simplement remettre en place les règles originales. Troisièmement, les processus de décision démocratique associés à certaines règles (ou à leur non-respect) sont beaucoup moins présents dans le wiki du cours. On pourrait ainsi soutenir que dans le wiki du cours, la communauté ne gère pas vraiment, ou très peu ces règles.

Mais ces différences sont moins grandes que ce qu'il peut en paraître de prime abord. D'une part, on a vu que dans Wikipédia, le fondateur Jimmy Wales s'est vu reconnaître implicitement une autorité, associée à la notion de « despote éclairé » (benevolent dictator; voir Reagle, 2011 : chap. 6). Si les règles détaillées ont été élaborées par la communauté, les principes de base de Wikipédia qu'il a proposés en 2001 n'ont jamais, eux, été remis en question (voir, par exemple «Wikipedia:Principes fondateurs »). D'autre part, les consignes et les règles peuvent être modifiées par tous les étudiants de la TÉLUQ, ce qui inclut les exétudiants du cours, qui n'ont plus à craindre une hypothétique répercussion sur leur résultat.

Il reste que le professeur, en vertu de sa responsabilité pédagogique et réglementaire, joue dans le wiki un rôle plus important et plus généralisé que tout individu dans Wikipédia. En ce sens, le wiki du cours se rapproche de ce que Bollier appelle un «commun sous garantie publique ». Il s'agit d'un commun pour lequel une instance (l'État, par exemple) joue un rôle de facilitateur, et garantit que règnent des « conditions équitables, non

discriminatoires » et que « les commoneurs disposent de l'autorité et de l'espace nécessaires pour s'engager dans un véritable "faire commun" ( Bollier, $2014: 42$ ).

Une dernière dimension doit cependant être considérée : la notion de gestion «pour le bien de tous » par une communauté. Dans Wikipédia, cela se traduit par l'offre d'une encyclopédie gratuite, bien sûr, mais aussi, complète et d'une qualité reconnue (malgré les réserves que l'on peut soulever à cet égard; voir Jullien, 2012 : 5.3.2), résultat de la taille et du dynamisme de sa communauté. De son côté, le wiki du cours SCI 1014, compte tenu du très petit nombre d'étudiants inscrits au cours et de l'absence, prévisible à la lumière des études précitées, d'autres contributions, n'est pas en mesure d'offrir au grand public qui y a accès une ressource riche et de qualité sur la science ouverte. De plus, la notion de communauté est ici réduite à sa plus simple expression : celle d'un ensemble (cumulatif) de personnes qui, actives en général à des périodes distinctes, ont peu d'interactions directes, mais sont reliées entre elles par des objectifs communs et une ressource à laquelle chacun 
ajoute ses contributions à ce que les participants précédents ont construit, et les offre aux « générations » futures.

\section{CONCLUSION}

Né de mon intention d'offrir à des étudiants la possibilité de se familiariser de manière concrète aux principes, valeurs et pratiques de la science ouverte, le cours SCI 1014 a pris la forme d'un wiki s'inspirant fortement de Wikipédia. Cependant, tant pendant la conception du cours et la mise au point du wiki que durant sa mise à l'essai, j'ai été amené à prendre diverses décisions faisant intervenir des enjeux éthiques. Ce processus de décision a été marqué par la tension entre, d'une part, la volonté de se rapprocher des conditions entourant la participation à Wikipédia et, d'autre part, le souhait de faciliter l'apprentissage chez les étudiants, le tout dans le respect des exigences liées à l'évaluation et la certification offertes par l'établissement. On peut considérer que le wiki du cours, du fait de sa similarité avec Wikipédia, répond formellement à la définition d'un commun, de type « sous garantie publique » compte tenu du rôle qu'y joue le professeur. Cependant, le très petit nombre d'étudiants ayant suivi le cours (deux ou trois par trimestre), et l'absence de contribution extérieure modifient le sens et la portée de la notion de communauté. Le modèle proposé ici pourrait cependant être repris dans un cours visant un auditoire plus important, avec une promotion effectuée pour susciter l'intérêt de l'ensemble des étudiants du programme, ou de l'établissement. Les conditions pour l'apparition d'une communauté vraiment interactive pourraient ainsi être réunies. Il serait alors possible d'effectuer une étude empirique, incluant notamment les points de vue des étudiants, sur la dynamique de ce commun voué à l'apprentissage et sur les conditions qui en favorisent le succès.

\section{REMERCIEMENTS}

Je tiens à remercier Julien Contamines pour les nombreuses discussions stimulantes survenues tout au long de la conception du cours et de la mise en place du wiki, de même que pour ses commentaires et suggestions sur une première version de ce texte. J'ai aussi bénéficié des explications éclairantes de Me Julie Carle sur les lois et règlements liés à l'éthique qui s'appliquent aux cours et aux environnements en ligne de la TÉLUQ. Je remercie enfin l'évaluateur anonyme qui, par ses commentaires judicieux, m'a permis de clarifier et d'approfondir certains aspects de l'argumentation.

\section{BIBLIOGRAPHIE}

BACHELET, Rémi (2008), « Mettre en œuvre un wiki académique », dans Actes du colloque Questions de pédagogie dans l'enseignement supérieur. En ligne à http://hal.archives-ouvertes.fr/halshs-00325678, consulté le 12 août 2015.

BAltZERSEN, Rolf K. (2010), «Radical Transparency: Open Access as a Key Concept in Wiki Pedagogy », Australasian Journal of Educational Technology, vol. 26, $\mathrm{n}^{\mathrm{o}}$ 6, p. 791-809.

BOLLIER, David (2014). La renaissance des communs : pour une société de coopération et de partage. Paris, Éditions Charles Léopold Mayer. 
CARDON, Dominique, et Julien LEVREL (2009), «La vigilance participative. Une interprétation de la gouvernance de Wikipédia », Réseaux, vol. 154, n 2, p. 51-89.

CARR, Nicholas (2011), «Questioning Wikipedia », dans Geert LOVINK et Nathaniel TKACZ (dir.), Critical Point of View: A Wikipedia Reader, Amsterdam, Institute of Network Cultures, p. 191-202.

Carroll, Michael W. (2013), "Creative Commons and the Openness of Open Access », New England Journal of Medicine, vol. 368, p. 789-791.

Every, Vanessa, Gna Garcia et Michael Young (2010), «A Qualitative Study of Public Wiki Use in a Teacher Education Program », dans David GIBSON et Bernie DoDGE (dir.), Proceedings of the Society for Information Technology \& Teacher Education International Conference (SITE), Chesapeake, VA, p. 55-62.

FLINN, Bill, et Hermann MAURER (1995), «Levels of Anonymity », Journal of Universal Computer Science, vol. 1, $\mathrm{n}^{\mathrm{o}}$ 1, p. 35-47.

Fountain, Renée (2005), «Wiki pedagogy », Dossiers technopédagogiques, Profetic. En ligne à http://www.crepuq.qc.ca/ancien/dossiers/spip.php?rubrique110, consulté le 15 août 2015.

GRIMMELMANN, James (2015), «The Virtues of Moderation », Yale Journal of Law \& Technology, vol. 17, p. 42-109.

GuTH, Sarah (2007), «Wikis in Education: Is Public Better? », dans Proceedings of WikiSym'07, ACM Press, p. 61-68.

JACQUEMIN, Bernard (2012), «Autorégulation de rapports sociaux et dispositif dans Wikipedia », Document numérique, vol. 14, n 3, p. 57-79.

JulliEN, Nicolas (2012), «What We Know About Wikipedia: A Review of the Literature Analyzing the Project(s) ». En ligne à http://hal.archives-ouvertes.fr/hal-00857208, consulté le 11 août 2015.

LiCHTENSTEIN, Sharman (2008), «The Wikipedia: Experts, Expertise and Ethical Challenges », dans Matthew WARrEN (dir.), AiCE 2008: Proceedings of the Fifth Australian Institute of Computer Ethics Conference, Melbourne, Deakin University, p. 112-118.

Mogin, Emmanuel (2010), «Perspectives - La science ouverte : un domaine en expansion », Conseil des académies canadiennes. En ligne à http://sciencepourlepublic.ca/fr/news/council-news/perspectives/dec2010.aspx, consulté le 25 août 2015.

MORRISON, Heather (2012). Freedom for scholarship in the internet age. Thèse de doctorat, Université Simon Fraser, Vancouver, Canada. En ligne à https://theses.lib.sfu.ca/thesis/etd7530, consulté le 15 août 2015.

MoshiRniA, Andrew V. (2007), «Important Ornaments: The Impact of Graphics and Rule Systems on Academic Wiki Use », dans Michael E. AUER (prés.), Proceedings of the Conference ICL2007. En ligne à http://www.iclconference.org/dl/proceedings/2007/paper/23_Final_Paper.pdf, consulté le 31 août 2015. 
NIELSEN, Michael (2011), Reinventing Discovery: The New Era of Networked Science, Princeton, Princeton University Press.

O’NEIL, Mathieu (2011), «Wikipedia and Authority », dans Geert LovINK et Nathaniel TKACZ, Critical Point of View: A Wikipedia Reader, Amsterdam, Institute of Network Cultures, p. 309-324.

Oz, Ayelet (2009), «Move Along Now, Nothing to See Here': The Private Discussion Spheres of Wikipedia », Social Science Research Network (SSRN). En ligne à http://papers.ssrn.com/sol3/papers.cfm?abstract_id=1726450, consulté le 15 août 2015.

REAGLE, Joseph Michael Jr. (2011), Good Faith Collaboration: The Culture of Wikipedia, Cambridge, MIT Press.

SAntAnA, Adele, et Donna J. Wood (2009), «Transparency and Social Responsibility Issues for Wikipedia », Ethics and Information Technology, vol. $11 \mathrm{n}^{\circ} 2$, p. 133-144.

SchneIDER, Daniel K., Kalliopi Benetos et Martine Ruchat (2011), «Mediawikis for research, teaching and learning », dans Theo BASTIAENS et Martin EBNER (dir.), Proceedings of World Conference on Educational Multimedia, Hypermedia and Telecommunications (EDMEDIA), Chesapeake, VA, AACE, p. 2084-2093.

Université du Québec à Montréal (n.d.), «Évaluer un site web », Infosphère. En ligne à http://www.infosphere.uqam.ca/analyser-linformation/evaluer-un-site-web, consulté le 24 août 2015.

WALES, Jimmy (2001), «Statement of principles », Wikipedia. En ligne à http://nostalgia.wikipedia.org/w/index.php?title=Jimbo_Wales/Statement_of_princip les\&oldid=75340, consulté le 12 août 2015.

Zheng, Binbin, Melissa NiIYA et Mark WARSCHAUER (2015), «Wikis and Collaborative Learning in Higher Education », Technology, Pedagogy and Education, vol. 24, $\mathrm{n}^{\circ} 3$, p. 357-374.

\section{Pages de Wikipédia}

http://en.wikipedia.org/wiki/Wikipedia:Expectations and norms of the Wikipedia community

http://fr.wikipedia.org/wiki/Wikipédia:Principes fondateurs

http://fr.wikipedia.org/wiki/Wikipédia:Travaux inédits

http://fr.wikipedia.org/wiki/Modèle:Tableau d'évaluation avancement

\section{Page de EduTech Wiki}

$\underline{\text { http://edutechwiki.unige.ch/en/EduTech_Wiki:Copyrights }}$

\section{Wiki du cours SCI 1014}

http://wiki.teluq.ca/SCI1014 